\title{
LOS MICRORRESTOS VEGETALES ACTUALES COMO HERRAMIENTA PARA LA RECONSTRUCCIÓN DEL USO DE LOS RECURSOS VEGETALES EN EL PASADO
}

\author{
Eliana F. Burgos ${ }^{1}$, María de los M. Colobig ${ }^{2}$ y Alejandro F. Zucol ${ }^{3}$ \\ Recibido: 20 de noviembre de 2017. Aceptado: 20 de marzo de 2018
}

\begin{abstract}
Resumen
El estudio de los granos de almidón y los esporomorfos recuperados en contextos arqueológicos sirve como una herramienta de investigación útil y novedosa que permite interpretaciones completas y evidencias directas sobre la interacción, domesticación, uso y consumo de las plantas en relación con los grupos humanos que habitaron un área determinada. El primer paso para identificar y contrastar estos restos arqueológicos es crear una colección de referencia distinguiendo y caracterizando aquellas plantas que son consideradas de importancia para la investigación. Existen registros desde la época prehispánica que hacen referencia al uso de especies pertenecientes a las familias Convolvulaceae y Fabaceae se usaban para la cura de enfermedades, con fines rituales y/o alimenticios. Ante esto, se seleccionaron especies nativas de la provincia de Entre Ríos pertenecientes a dichas familias con el objeto de estudiar tanto los granos de almidón como las características palinológicas de las mismas. Para su análisis, se recolectaron tres ejemplares por especie y se extrajeron granos de almidón de la raíz empleando métodos convencionales y esporomorfos mediante colecta en fresco. Los resultados obtenidos proporcionan información de importancia para identificar y contrastar los almidones hallados en contextos arqueológicos de la región.
\end{abstract}

Palabras clave: palinología, granos de almidón, plantas medicinales, colección de referencia.

\begin{abstract}
The use of starch and pollen grains in archaeological contexts is an useful and innovative research tool; that allows complete interpretations and direct evidence of the interaction, domestication, use and consumption of the plants related to human groups that inhabited a determined place. The first step to make their identification in archaeological environmental is the reference collection of local plant material allowing the identification of found materials. Convolvulaceae and Fabaceae species were recorded in pre-Hispanic populations with ritual and food purpose. In this research, native species of these families present in Entre Rios province were study in their starch grains and palynological features. The starch and pollen elements were extracted by conventional methods, and their analysis provide important information to characterize them, which would allow to be used in future archaeological studies.
\end{abstract}

Key words: palynology, starch grain, medicinal plant, reference collection.

1 Facultad de Ciencia y Tecnología, U.A.D.E.R. Km 10.5 RP11, Oro Verde, Entre Ríos. E-mail: burgosef@gmail.com

2 Facultad de Ciencia y Tecnología, U.A.D.E.R. Km 10.5 RP11, Oro Verde, Entre Ríos. Laboratorio de Arqueología, Centro de Investigaciones Científicas y Transferencia de Tecnología a la Producción (CICYTTP-UADER - Provincia de Entre Ríos - CONICET), Dr. Materi y España SN, E3105BWA, Diamante, Argentina. E-mail: milagroscolobig@gmail.com

3 Facultad de Ciencia y Tecnología, U.A.D.E.R, Km 10.5 RP11, Oro Verde, Entre Ríos. CICYTTP-CONICET, España y Matteri s/n, Diamante, Entre Ríos. E-mail: cidzucol@infoaire.com 


\section{Introducción}

Las plantas que poseen en su metabolismo principios activos utilizados por su acción farmacológica y beneficiosa para los organismos vivos pueden denominarse como plantas medicinales (Muñoz 1996). La acción humana puede modificar las comunidades vegetales a través de actividades como el uso, cultivo, protección y/o el transporte de estas plantas al migrar los grupos humanos. Esto ha producido que ciertas especies se distribuyan en otras regiones y que aumenten la variación composicional de las comunidades vegetales (Colobig et al. 2015).

La confección de colecciones de referencia constituye un primer paso en toda investigación paleoetnobotánica. A partir de estas colecciones es posible comparar microrrestos vegetales hallados en contextos arqueológicos con la vegetación actual y de esta forma inferir si se trata de especies nativas, si fueron introducidas u obtenidas de otras regiones.

Los microrrestos vegetales son distintos tipos de partículas microscópicas de origen vegetal, tales como gránulos de almidón, silicofitolitos, fitolitos de calcio, granos de polen, esporas, que constituyen inclusiones celulares, partículas intercelulares y órganos (Babot 2001). Su producción diferencial está controlada genéticamente, por lo que las características morfométricas y propiedades ópticas resultantes poseen valor taxonómico (Esau 1976; Loy 1994: 28; Mulholland y Rapp 1992; Babot 2007).

Los granos de almidón se componen principalmente por dos polimeros: amilosa (soluble) y amilopectina (insoluble) (Radley 1943). Se encuentran principalmente en las raíces, tubérculos, semillas y órganos de reserva, y generalmente las diferentes especies de plantas producen sus propias formas de granos (Yang y Jiang 2010). El estudio de sus caracteres morfológicos permite elaborar catálogos donde se des- criben aquellos rasgos diagnósticos que facilitan la identificación taxonómica de las especies que lo producen (Vargas 2004).

Los granos de polen y esporas, por su parte, tienen una forma básica que puede variar en su tamaño; a estas morfologías se les suma la ornamentación de la pared y estructuras como sacos aéreos y características exínicas, que por lo general cumplen un papel ecológico, y a su vez, constituyen caracteres distintivos de cada taxón. Al estar estas estructuras involucradas en la reproducción de la planta, poseen aberturas por donde emerge el contenido celular durante la fecundación, llamados poros y sulcos, de acuerdo a su forma. Estos constituyen, al igual que otras estructuras morfológicas, caracteres diagnósticos para su identificación. Los granos de polen permiten identificar qué planta los produce y sabiendo sus características mesológicas se puede inferir características del clima de una determinada área, como humedad, épocas frías y cálidas; así como también conocer las variaciones composicionales de la flora y rutas de avance de la vegetación o de los grupos humanos (de Oliveira Freitas 2002).

La región del Delta del Paraná es potencialmente rica en sitios arqueológicos, como lo evidencian las prospecciones en las que se han detectado más de 100 sitios, con ocupaciones del Holoceno tardío (últimos 2000 años) (Bonomo et al. 2010; Colobig et al. 2015). Sin embargo, la información con que se cuenta sobre el alcance diagnóstico de los caracteres de almidones y polen en plantas nativas de uso medicinal de la provincia de Entre Ríos es escasa.

Mediante el análisis morfométrico y morfológico de los microrrestos antes mencionados se puede establecer cuáles poseen valor sistemático. Debido a ello el objetivo de este trabajo es el análisis y la caracterización de dos tipos de microrrestos de origen vegetal, granos almidón y polen, provenientes de cinco especies vege- 
tales actuales (colectadas en la ciudad de Diamante) que se vinculan con los pueblos originarios de la zona, ya sea este uso documentado por fuentes directas o indirectas. Tal es el caso de Ipomoea cairica (L.) Sweet, que ha sido citada para preparación de infusiones de hojas contra afecciones pulmonares, infusiones diuréticas en enfermedades renales e hinchazón, así como para tratamientos por reumatismo (Ferreira et al. 2006; Meira et al. 2012), purgante, febrífugas, antihidrópicas y vulnerarias en fomentos y sus flores se han utilizado en fumatas con fines rituales (Lahitte et al. 1998). Para Ipomoea indica (Burm.f) Meer los registros muestran la utilización en triturados o decoctados como remedio sobre mordeduras de serpientes (Hieronymus 1882), también usado como purgante y para curación de fractura de huesos (Abbott y Shimazu 1985; Meira et al. 2012). Diferentes autores asignan a las especies Acacia caven (Molina) Molina y Erythrina crista-galli L. propiedades astringentes, antisépticas, antiasmáticas y para combatir la tos (Hieronymus 1882; Lahitte et al. 1998; Rondina et al. 2008); mientras que para la especie Parkinsonia aculeata L. las citas indican su utilización como diaforéticas, febrífugas, abortivas y antipalúdica (Hieronymus 1882; Rondina et al. 2008).

El análisis de microrrestos de las especies mencionadas tiene como finalidad la confección de una colección de referencia que permita en estudios posteriores llevar adelante comparaciones con material proveniente de sitios arqueológicos.

\section{Materiales y Métodos}

Se analizaron los granos de almidón y polen de cinco especies vegetales nativas (Tabla 1). Los ejemplares estudiados fueron recolectados durante el año 2014 en el departamento Diamante, Provincia de Entre Ríos, el cual se encuentra inmerso en la Ecorregión del Delta e Islas del Paraná (Burkart et al. 1999). Esta ecorregión está caracterizada por la confluencia de diferentes regiones fitogeográficas por lo que presenta alta diversidad y riqueza de especies vegetales (Cabrera y Willink 1980). Los ejemplares herborizados se encuentran depositados en el Laboratorio de Paleobotánica del CICYTTP-UADER - Provincia de Entre Ríos - CONICET.

\section{Extracción de granos de almidón}

Para extraer los granos de almidón, se cortó la raíz de las especies del género Ipomoea, mientras que para las leguminosas se utilizó el fruto en estado inmaduro y maduro. Se raspó el órgano seleccionado con aguja histológica y con bisturí sobre el portaobjeto y se montaron en glicerina, para que de esta forma, las micropartículas desagregadas se preserven inalteradas y móviles, a fin de apreciar con claridad sus atributos distinguibles. Los granos de almidón se examinaron y fotografiaron con luz polarizada y normal a 40x. Se consideraron tres ejemplares por especie a partir de los cuales se obtuvo un total de cuatro muestras por ejemplar. Los caracteres que se observaron en los granos de almidón fueron: tamaño y forma, características del hilum, lamela y cruz de extinción, contornos y bordes, y si son granos compuestos o simples (Babot 2001; Babot 2007; Cortella y Pochettino 1994, 1995; Musaubach 2013). Se obtuvieron cuatro muestras por ejemplar a partir de las cuales se pudo observar y caracterizar 365 granos de almidón para I. indica y 390 para I. cairica; mientras que para las especies de Fabaceae los granos de almidón identificables fueron escasos.

\section{Extracción de granos de polen}

Para el caso de los granos de polen, se utilizaron anteras provenientes de material 


\begin{tabular}{|c|c|c|c|c|}
\hline \multicolumn{2}{|c|}{} & $\begin{array}{c}\text { Lugar y fecha de } \\
\text { recolección }\end{array}$ & Colector & Herbario \\
\hline Convolvulaceae & $\begin{array}{c}\text { Ipomoea } \\
\text { indica } \\
\text { (Burm. f.) }\end{array}$ & Diamante, 2014 & Burgos, Eliana & $\begin{array}{c}\text { Laboratorio de } \\
\text { Paleobotánica, } \\
\text { CICyTTP- } \\
\text { CONICET }\end{array}$ \\
\cline { 2 - 5 } & $\begin{array}{c}\text { Ipomoea } \\
\text { cairica (L.) }\end{array}$ & Diamante, 2014 & Burgos, Eliana & $\begin{array}{c}\text { Laboratorio de } \\
\text { Paleobotánica, } \\
\text { CICyTTP- } \\
\text { Fabaceae }\end{array}$ \\
& $\begin{array}{c}\text { Erythrina } \\
\text { crista-galli } \\
\text { (L.) }\end{array}$ & Diamante, 2014 & Burgos, Eliana & $\begin{array}{c}\text { Laboratorio de } \\
\text { Paleobotánica, } \\
\text { CICyTTP- } \\
\text { CONICET }\end{array}$ \\
\cline { 2 - 5 } & $\begin{array}{c}\text { Parkinsonia } \\
\text { aculeata } \\
\text { (L.) }\end{array}$ & Diamante, 2014 & Burgos, Eliana & $\begin{array}{c}\text { Laboratorio de } \\
\text { Paleobotánica, } \\
\text { CICyTTP- } \\
\text { CONICET }\end{array}$ \\
\cline { 2 - 5 } & $\begin{array}{l}\text { Acacia } \\
\text { caven } \\
\text { (Molina) } \\
\text { Molina }\end{array}$ & Diamante, 2014 & Burgos, Eliana & $\begin{array}{c}\text { Laboratorio de } \\
\text { Paleobotánica, } \\
\text { CICyTTP- } \\
\text { CONICET }\end{array}$ \\
\hline
\end{tabular}

Tabla 1. Especies vegetales nativas utilizadas en este trabajo.

fresco que fueron cortadas y colocadas en tubos eppendorf con $1 \mathrm{ml}$ de agua destilada. Se agregó una gota de safranina para la coloración y se llevó a centrífuga por 15 minutos a $2.500 \mathrm{rpm}$. Luego se extrajo el sobrenadante y se montó en un portaobjeto con glicerina para su posterior observación microscópica. Se utilizó un microscopio óptico Arcano y se fotografiaron a 40x y 100x. Se observaron las características de la exina, ornamentación, presencia y tamaño de colpos o poros. Se obtuvieron tres muestras por ejemplar a partir de las cuales se pudieron observar y caracterizar entre 50 y 60 granos de polen por especie.

\section{Resultados}

\section{Granos de almidón}

Ipomoea indica. Granos de almidón de formas esféricas principalmente, ovoides o reniformes (Figura 1 A-A', B-B'), de tamaños medios de 9,59 $\mu \mathrm{m}$ de ancho y 8,71 $\mu \mathrm{m}$ de largo. El hilum no se encuentra claramente visible. Granos afisurados y alamelados. La cruz de extinción es céntrica, con brazos simétricos a levemente curvados. En algunos casos se observaron granos con cruz excéntrica (Tabla 2). No son visibles 


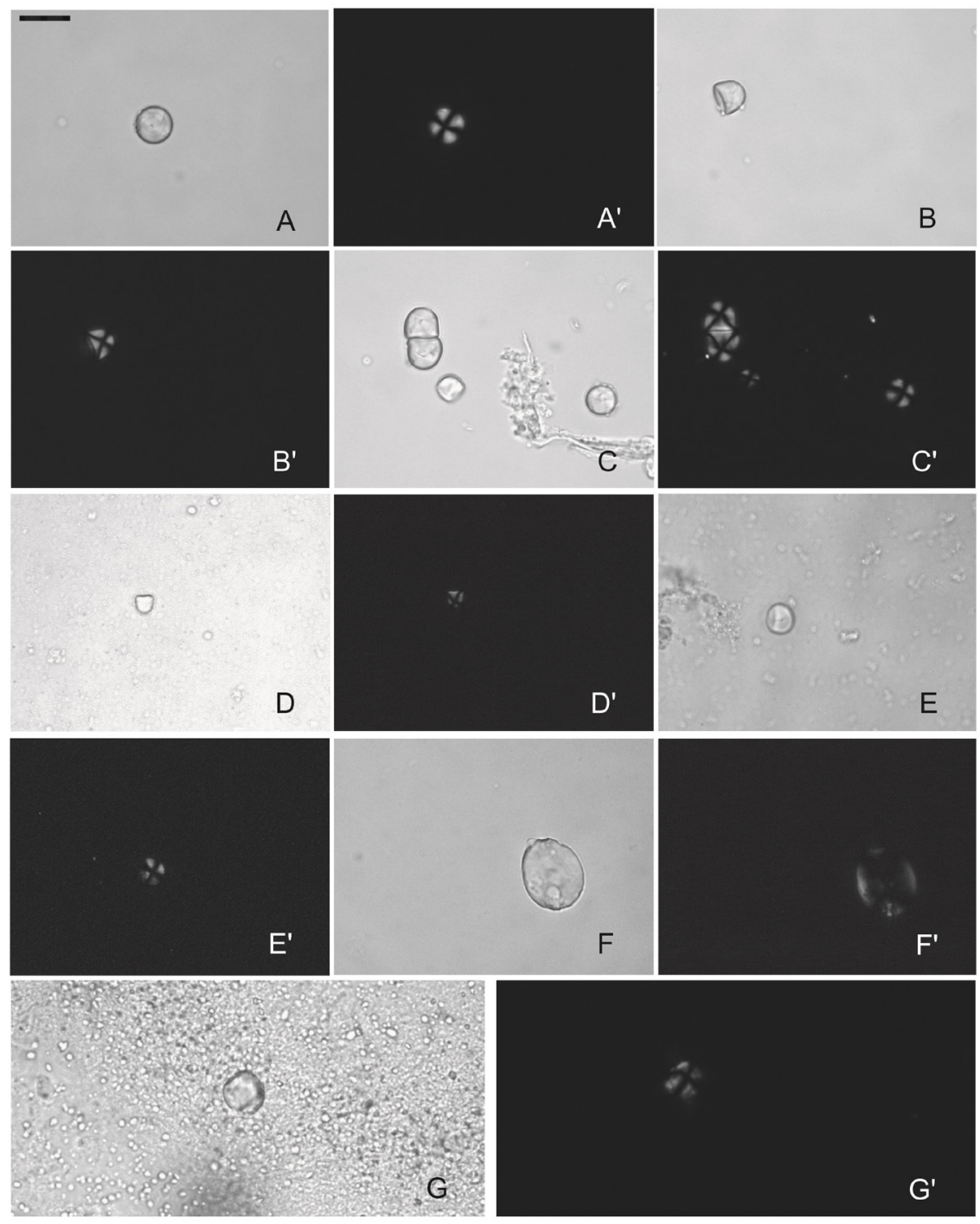

Figura 1. A-A': grano de almidón simple de Ipomoea indica, de forma esférica con bordes regulares, lamelas incompletas, fisura céntrica, hilo invisible, con cruz de extinción céntrica y simétrica. B-B': grano de almidón simple de $I$. indica de forma acampanada con bordes regulares, alamelado, afisurado, hilo invisible, con cruz de extinción excéntrica de brazos levemente curvados y simétricos. C-C': grano de almidón compuesto de $I$. indica. D-D': Grano simple de Ipomoea cairica, de forma acampanada con bordes regulares, alamelados, afisurados, con hilo invisible, con cruz de extinción céntrica levemente excéntrica con brazos simétricos. E-E': grano de almidón simple de $I$. cairica de forma ovalada, afisurado, alamelado, con hilum invisible, y cruz de extinción céntrica con brazo asimétricos. F-F': Estructuras celulares halladas en semillas secas de Acacia caven. G-G': grano de almidón simple de Erythrina cristagalli., esférico alamelado y afisurado, con hilo invisible y cruz de extinción céntrica con brazos irregulares. Observaciones hechas en microscopio óptico con luz normal y luz polarizada en 40x10. Escala de $20 \mu \mathrm{m}$ en A. 


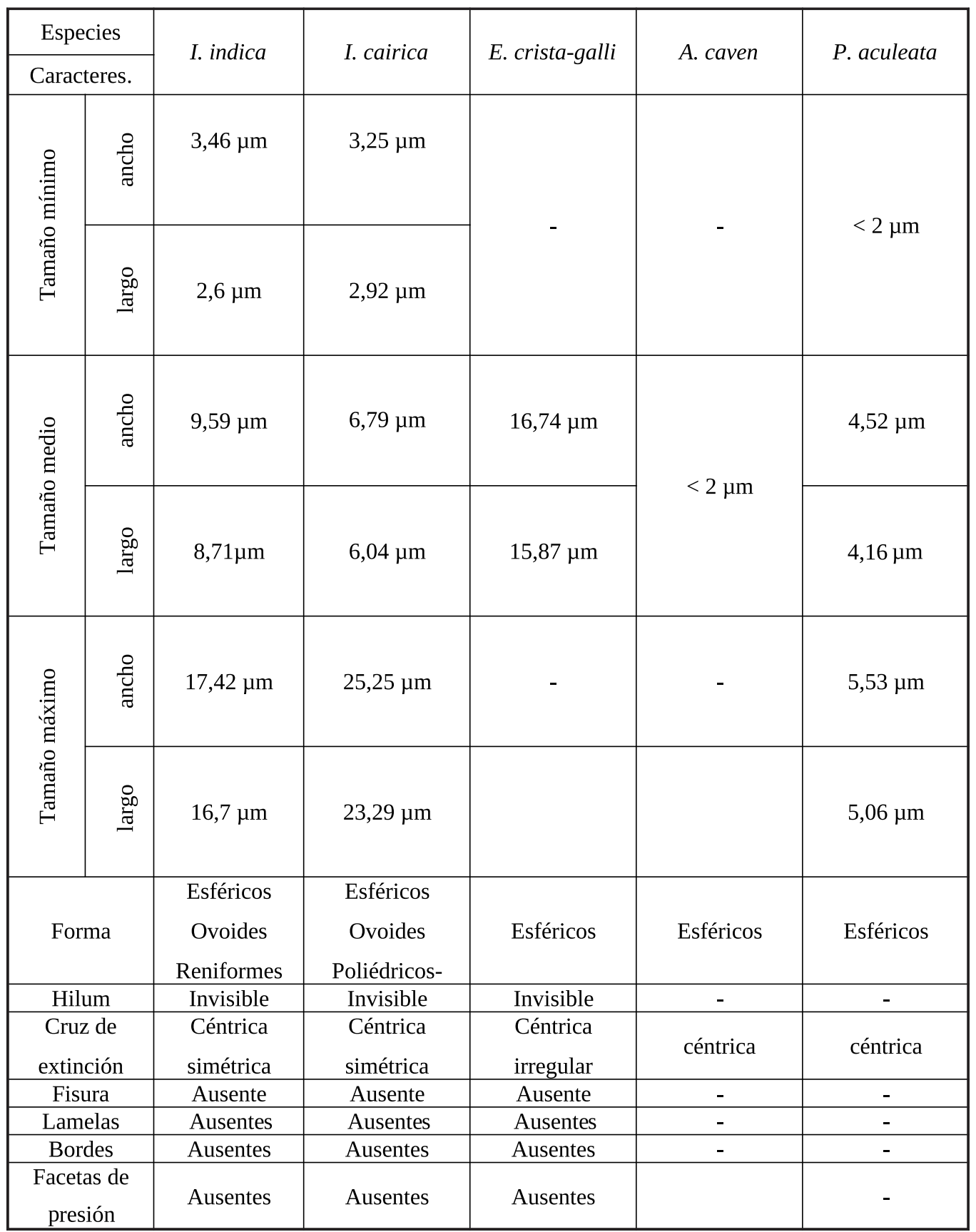

Tabla 2. Caracteres de los granos de almidón de las especies tratadas. 
facetas de presión, bordes irregulares o rebordes. Son granos mayormente simples pero se los puede hallar formando grupos de numerosos granos con poca separación, constituyendo diadas, tríadas o racimos (Figura.1 C-C'). Se hallaron anillos de celulosa.

Ipomoea cairica. Granos de almidón de formas mayormente esféricas, ovoides o poliédricas, aunque se observan con frecuencia formas de tipo prismáticas o conoides (Figura 1 D-D', E-E'), de tamaños medios de 6,79 $\mu \mathrm{m}$. El hilum se presenta invisible, rara vez visible y céntrico. Granos predominantemente afisurados y en ocasiones con fisuras radiales. No se observan lamelas y las cruces de extinción son céntricas de brazos asimétricos (Tabla 2). Se observaron células con almidones en su citoplasma.

Acacia caven. Granos de almidón menores a $2 \mu \mathrm{m}$ en los cuales no fue posible observar las características relevantes. En las muestras obtenidas a partir de las semillas del año 2014 se observó una sola estructura de forma circular que posee en su interior estructuras semejantes a la cruz de extinción y facetas, lo que podría indicar ser un grano compuesto amorfo (Tabla 2).

Parkinsonia aculeata. Granos de almidón con tamaño medio de 4,52 $\mu \mathrm{m}$ de ancho y 4,16 $\mu \mathrm{m}$ de largo, tamaños mínimos menores a $2 \mu \mathrm{m}$ y máximos de 5,53 $\mu \mathrm{m}$ de ancho y 5,06 $\mu \mathrm{m}$ de largo. Se observa la presencia de numerosos granos de almidón de tamaños menores a $2 \mu \mathrm{m}$. En ningún caso fue posible observar los caracteres que los definen a excepción de la cruz de extinción céntrica. En su mayoría son granos compuestos formando diadas o tríadas (Tabla 2).

Erythrina cristagalli. Para el caso de las semillas inmaduras se encontró un solo grano de almidón simple, sólido, de forma esférica, alamelado, afisurado y con hilum invisible (Figura 1 G-G'). La cruz de extinción se presenta en forma céntrica irregular, con uno de sus brazos quebrado. Se observan también irregularidades en la superficie del grano que pueden asociarse a facetas de presión (Tabla 2).

\section{Palinologia}

Ipomoea indica. Grano de polen en mónades (Figuras 2 y 3); apolar; radiosimétrico o asimétrico; escábrido; exina de 12,58 $\mu \mathrm{m}$ de espesor, intectada, se observan las columelas, sexina y nexina distinguibles; equinado con espinas de base ancha de

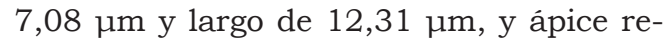
dondeado; polipantoporados; granos esferoidales o prolado-esferoidales, relación $\mathrm{P} / \mathrm{E}=1,03 \pm 0,025$; muy grandes, tamaños medios de $131,71 \mu \mathrm{m}$ de ancho y 128,18 um de largo (Tabla 3).

Ipomoea cairica. Grano de polen en mónades (Figuras 4 y 5); apolar; radiosimétrico o asimétrico; escábrido; exina de 4,96 $\mu \mathrm{m}$ de espesor, sexina e intina delgadas sin poder diferenciarse una de otra; equinado con espinas de base ancha de 5,58 $\mu \mathrm{m}$ de diámetro y largo 11,31 $\mu \mathrm{m}$, ápice aguzado; polipantoporados; granos esferoidales o prolado-esferoidales, relación $\mathrm{P} /$ $\mathrm{E}=1,03 \pm 0,017$; grandes, de tamaño medio de 99,46 $\mu \mathrm{m}$ de ancho y 96,61 $\mathrm{m}$ de largo (Tabla 3).

Acacia caven. (Tabla 3) Grano de polen en poliades ovoides; isopolar; psilado; exina de 1,34 $\mu \mathrm{m}$; de espesor inaperturado; semitectado; granos subprolados o prolados alargados, relación $\mathrm{P} / \mathrm{E}=1,24 \pm 0,24$; medianos a grandes; tamaño medio de la asociación 38,08 $\mu \mathrm{m}$ de ancho y 31,61 $\mu \mathrm{m}$ de largo (Figura 6). 

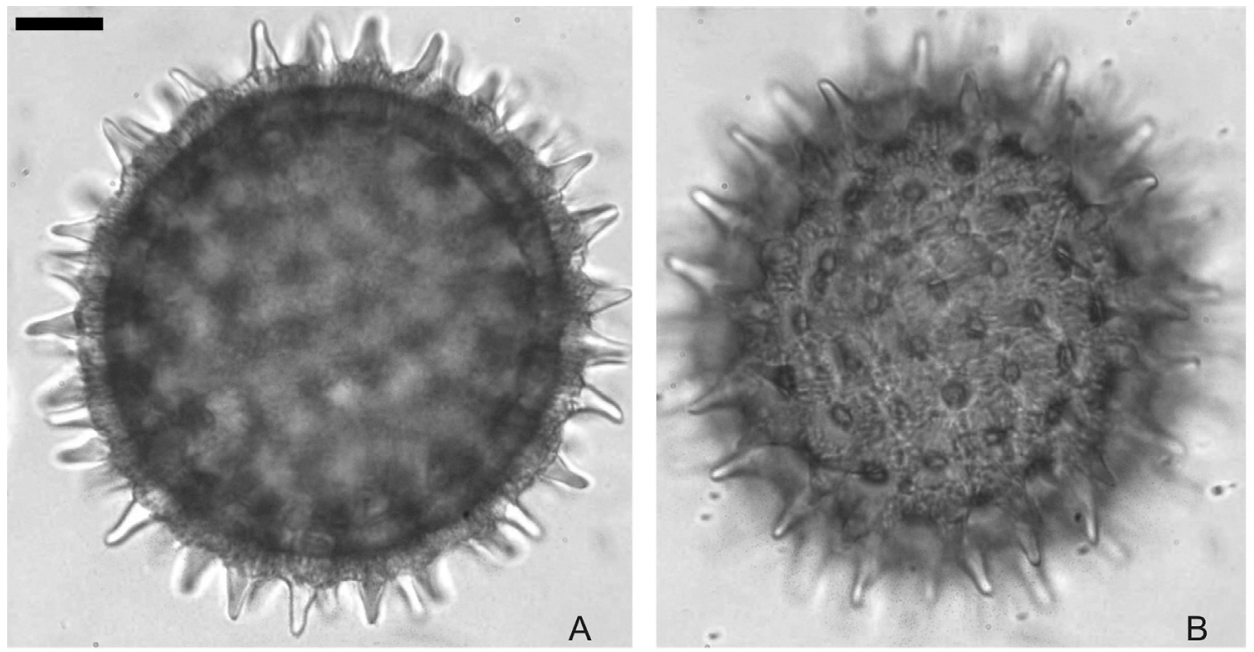

Figura 2. Vista del grano de polen de $I$. indica en corte óptico (A) y vista superficial (B). Vista en 40x. Reglilla en A (20 $\mu \mathrm{m})$.
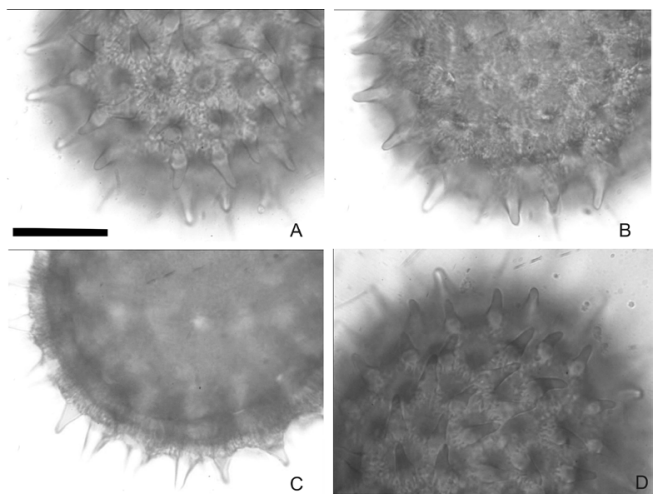

Figura 3. Grano de polen de I. indica vista en 100x. En A, B y D se observa la exina irregular, espinosa y aperturas. En C, corte óptico donde se marca con una flecha las columnelas de la exina y las espinas. Reglilla en A $(20 \mu \mathrm{m})$.
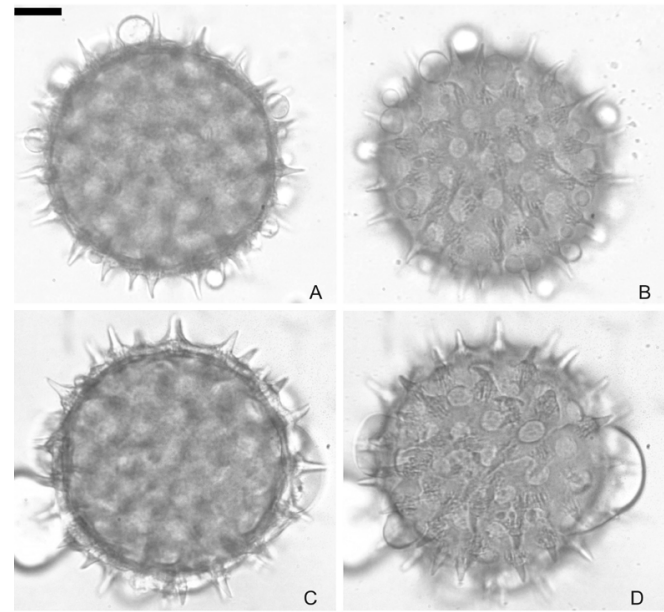

Figura 4. Granos de polen de I. cairica en corte óptico (A y C) y esculturas de la exina (B y D), vista en 40x. Reglilla en A $(20 \mu \mathrm{m})$. 

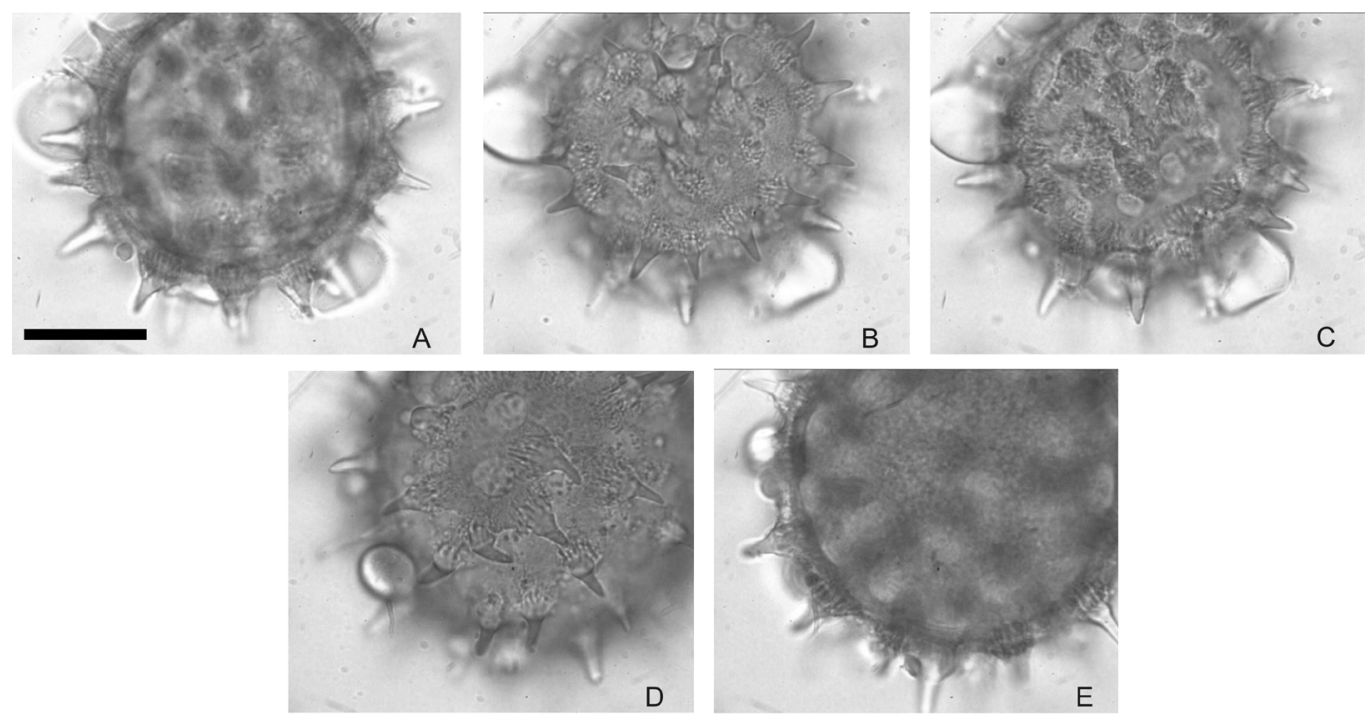

Figura 5. Granos de polen de I. cairica vistas en 100x. En A y E se observa el corte óptico. En B, C y D se observan las esculturas espinosas de la exina. Reglilla en A (20 $\mu \mathrm{m})$.
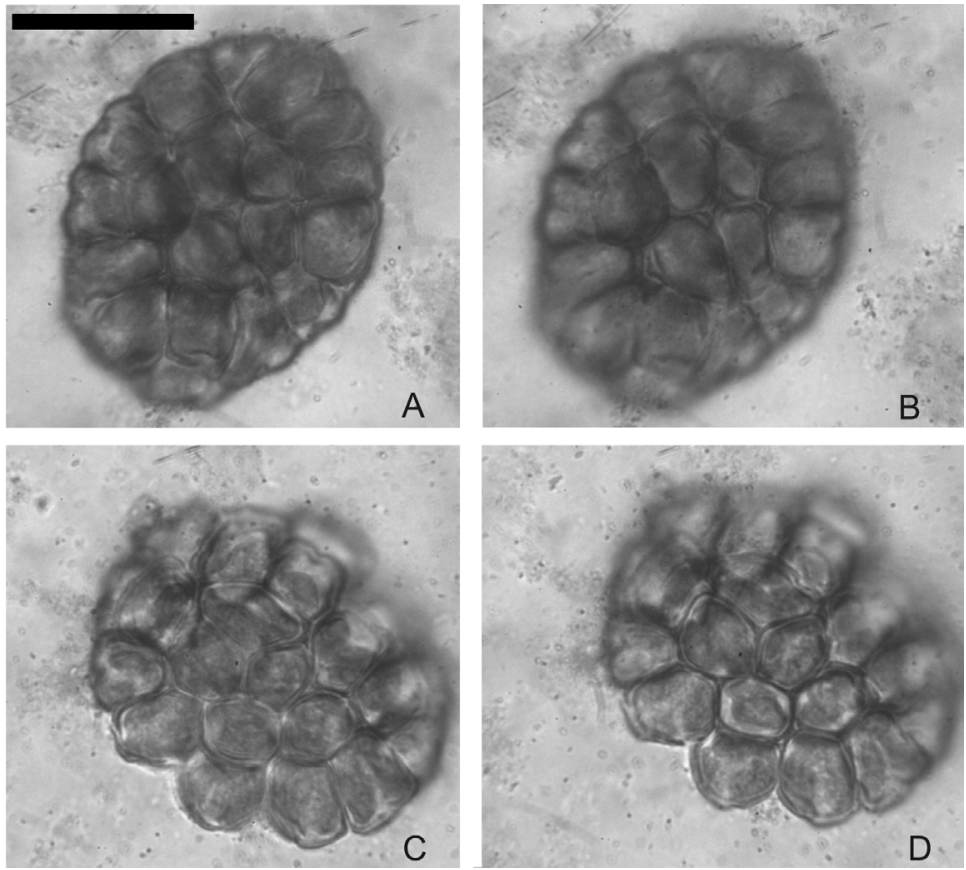

Figura 6. Poliadas de A. caven en vista ecuatorial vistas en A- D a 100x. Reglilla en A (20 $\mu \mathrm{m})$. 

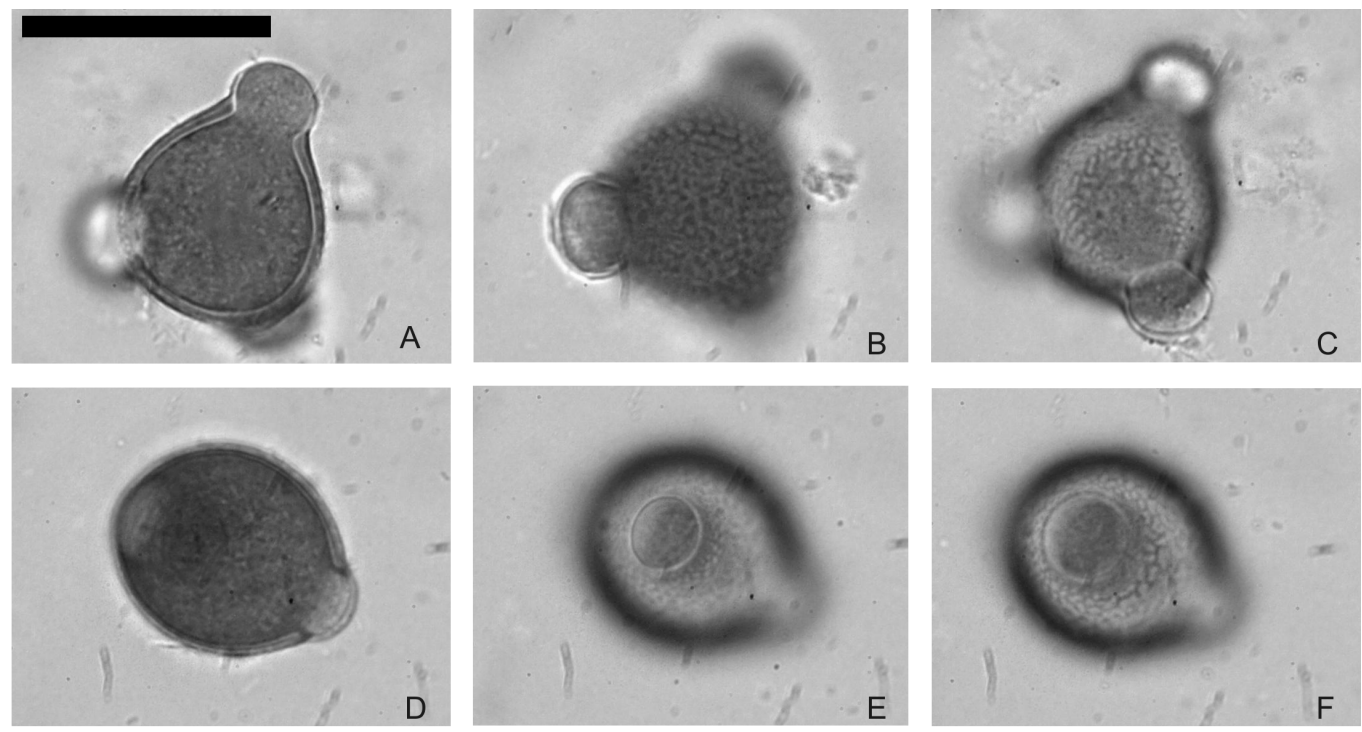

Figura 7. Granos de polen de E. cristagalli vistos en 100x. A-B: granos de polen en vista polar. Se observa exina reticulada y poros. D-E: granos en vista ecuatorial. Reglilla en A (20 $\mu \mathrm{m})$.

Erythrina critagalli. (Tabla 3) Granos de polen en mónades, heteropolares, radiosimétricos; reticulados con lúmenes irregulares; exina 1,41 $\mu \mathrm{m}$ de espesor; tripororado (poros+endoabertura); operculado: poro circular de 8,21 $\mu \mathrm{m}$ de diámetro, anulus de 1,35 $\mu \mathrm{m}$ de diámetro; tectado; trilobado; granos esferoidales, prolado-esferoidal o subprolados, relación $\mathrm{P} / \mathrm{E}=1,15 \pm 0,10$; granos pequeños a medianos; tamaños medios de $29,10 \mu \mathrm{m}$ de ancho y $25,92 \mu \mathrm{m}$ de largo (Figura 7).

Parkinsonia aculeata. (Tabla 3) Granos de polen en mónades, heteropolares, radiosimétricos; reticulados con lúmenes irregulares, psilado cercano a los poros; tectado; exina de 0,95 $\mu \mathrm{m}$ de espesor; tricolporado, endoaperturado; trilobado; granos esferoidales o prolado-esferoidales, relación $\mathrm{P} / \mathrm{E}=1,09$; pequeños; tamaños medios de $18,37 \mu \mathrm{m}$ de ancho y $16,87 \mu \mathrm{m}$ de largo (Figura 8).

\section{Discusiones}

Siguiendo el esquema de exposición de los resultados, para las especies del género Ipomoea, (I. indica e I. cairica), se observó que las características de los granos de almidón no permiten establecer una diferenciación taxonómica entre estas especies, como sí lo permiten los almidones característicos de la especie Ipomoea batatas (Korstanje y Babot 2005) en la que los granos provenientes de tubérculos presentan formas acampanadas, ovoides o poliédricas, con presencia de facetas, hilum excéntrico y distintivo, presencia de lamelas y cruz de extinción excéntrica. En el caso de las convolvulaceas estudiadas, las diferencias pueden observarse en cuanto al tamaño medio, pero los rangos de variabilidad del tamaño no nos permiten asegurar que éste carácter sea diagnóstico.

Para el caso de la palinología de I. indica e $I$. cairica la bibliografia es escasa. Aun así 

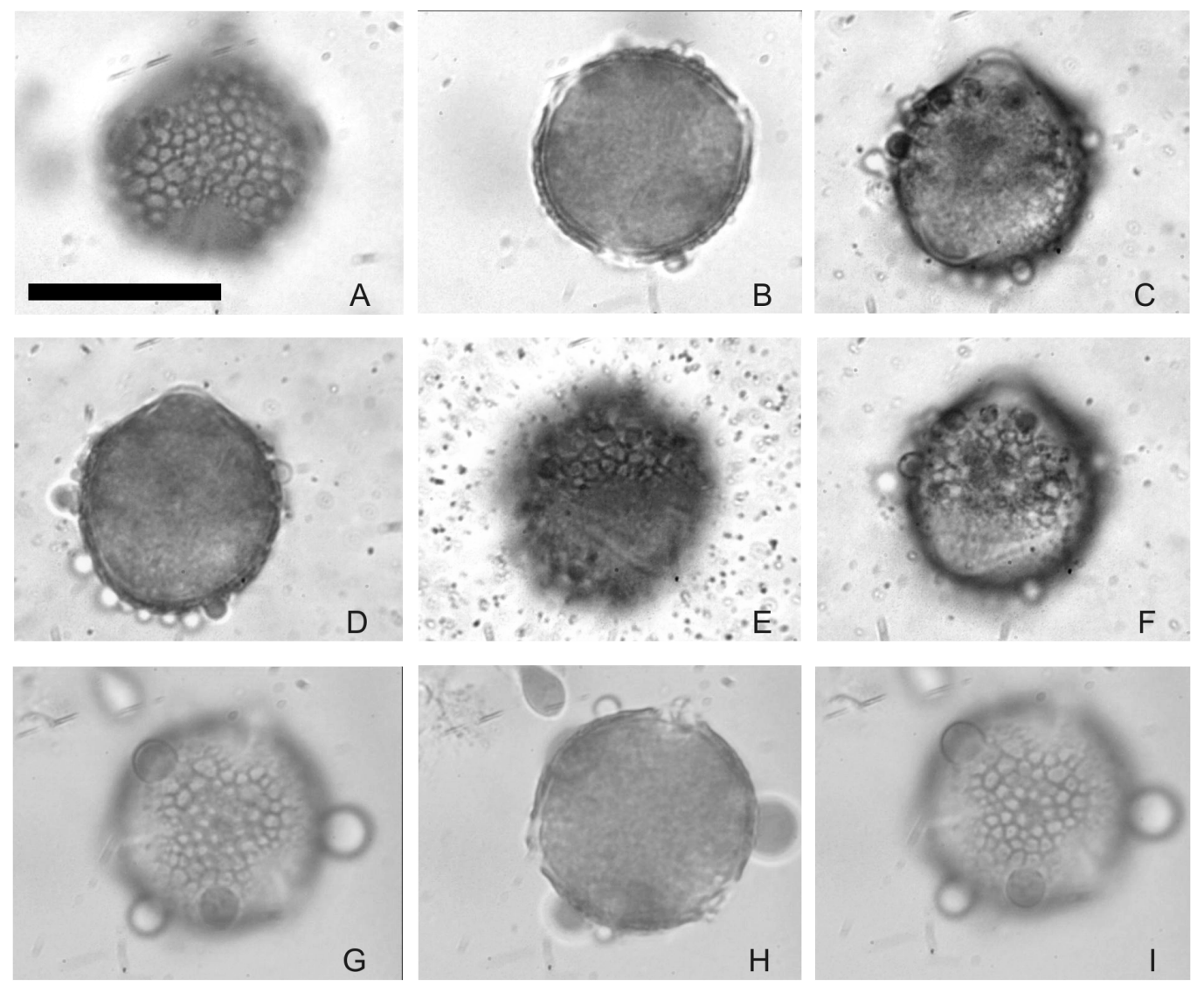

Figura 8. Grano de polen de P. aculeata en vista polar (A-B; G-I); en vista ecuatorial (C-F). Vista en 100 x. en E y F se observa la abertura colporada caracteristica del grano. Reglilla en A (20 $\mu \mathrm{m})$.

ha sido posible establecer diferencias entre éstas, partiendo del tamaño del grano (I. indica posee granos de mayor tamaño que I. cairica), así como también se diferencian en el tamaño de los elementos supratectales, grosor de la exina y diferenciación de sexina/nexina vista al microscopio óptico; teniendo como similitudes exina irregular y polipantoporada. Ambas especies comparten características con especies presentes en la zona como I. nil (Sanches-Dzib et al. 2009) e I. purpurea (Faye et al. 2002).
Las leguminosas analizadas presentaron abundantes almidones de transición en los frutos inmaduros, y escasos almidones con caracteres distinguibles.

En el estudio palinológico se comprobó que la técnica utilizada es apta para el análisis morfométrico de los granos de polen de las especies tratadas. Los resultados obtenidos concuerdan con los estudios previos llevados a cabo por diferentes autores (Markgraf y D’Antoni 1978; Díaz de la Guardia et al. 1988; Faricelli et al. 


\begin{tabular}{|c|c|c|c|c|c|}
\hline Especies & \multirow[b]{2}{*}{ I. indica } & \multirow[b]{2}{*}{ I. cairica } & \multirow[b]{2}{*}{ E. crista-galli } & \multirow[b]{2}{*}{ A. caven } & \multirow[b]{2}{*}{ P. aculeata } \\
\hline Caracteres & & & & & \\
\hline Granos & Mónade & Mónade & Mónade & Políada & Mónade \\
\hline Polaridad & Apolar & Apolar & Heteropolar & Isopolar & Heteropolar \\
\hline Simetría & Radiosimétrico & Radiosimétrico & Radiosimétrico & - & Radiosimétrico \\
\hline $\begin{array}{l}\text { Espesor de } \\
\text { exina }(\mu \mathrm{m})\end{array}$ & 12,58 & 4,96 & 1,41 & 1,34 & 0,95 \\
\hline Ornamentación & \multicolumn{2}{|c|}{ Equinada } & psilados & psilados & psilados \\
\hline Sist. Apertural & \multicolumn{2}{|c|}{ Polipantoporado } & $\begin{array}{l}\text { Tripororado } \\
\text { operculado }\end{array}$ & Inaperturado & Tricolporado \\
\hline Forma & \multicolumn{2}{|c|}{ Esféroidal/prolado esferoidal } & $\begin{array}{l}\text { Esferoidal, } \\
\text { prolado } \\
\text { esferoidal o } \\
\text { subprolado }\end{array}$ & $\begin{array}{c}\text { Subprolado/ } \\
\text { alargado }\end{array}$ & $\begin{array}{l}\text { Trilobado, } \\
\text { esferoidal }\end{array}$ \\
\hline Tamaño & Muy grandes & Grandes & $\begin{array}{l}\text { Pequeños- } \\
\text { medianos }\end{array}$ & $\begin{array}{c}\text { Medianos- } \\
\text { grandes }\end{array}$ & Pequeños \\
\hline
\end{tabular}

Tabla 3. Características de los palinomorfos de las especies tratadas.

2004; Campos-Trujillo et al. 2015). En el caso de la especie $A$. caven el tamaño difiere del obtenido por autores como Faricelli et al. (2004) que data en $66,07 \pm 6,61 \mu \mathrm{m}$; coincidiendo en caracteres como exina psilada y políadas constituidas por más de 30 células.

\section{Conclusiones}

Los resultados expuestos evidencian la presencia de granos de almidón en diversas morfologías en la familia Convolvulaceae y escasa presencia de microrrestos almidonosos en la familia Fabaceae. Bajo estas perspectivas y resultados, es posible la utilización de los granos de almidón de las especies $I$. indica e $I$. cairica como elementos de diferenciación taxonómica a nivel especie siempre que se encuentren acom- pañados de palinomorfos, mientras que si se los considera como partículas aisladas, pueden ser utilizados para caracterizaciones a nivel de género o familia. En el caso de las especies de leguminosas, se sugiere realizar estudios integrales que abarquen otras estructuras de almacenamiento de almidón en estas especies debido a que predominaron almidones transitorios que no constituyen caracteres diagnósticos.

En cuanto a los granos de polen los resultados obtenidos concuerdan con la bibliografia consultada y aportan al enriquecimiento de la información local sobre las especies tratadas.

Es necesario ampliar estos conocimientos y ahondar en la búsqueda de nuevos caracteres que nos permitan diferenciar las especies con el uso de microrrestos no palinológicos, así como también evaluar la palinología de especies relacionadas en 
la región. De esta manera, el espectro de elementos con carácter diagnóstico podrá acrecentarse y constituir una herramienta cada vez más certera de reconstrucción no sólo paleobotánica, sino etnológica y antropológica, donde se pueda inferir el uso, disponibilidad, domesticación y evolución etnobotánica de los recursos vegetales utilizados por los pueblos originarios de la región del Delta del Paraná.

\section{Bibliografia}

ABBOTT, I.A. y C. SHIMAZU. 1985. The geographical origin of plants most commonly used for medicine by Hawaiians. Journal of Ethnopharmacology 14: 213222.

BABOT, M.P. 2001. Almidones y fitolitos: desentrañando el papel funcional de los artefactos de molienda arqueológicos. En Arqueología argentina en los inicios de un nuevo siglo, editado por F. Oliva, N. de Grandis y J. Rodíguez, pp. 665717. Laborde, Rosario.

BABOT, M.P. 2007. Granos de almidón en contextos arqueológicos: posibilidades y perspectivas a partir de casos del Noroeste argentino. En Paleoetnobotánica del Cono Sur: estudios de casos y propuestas metodológicas, editado por $\mathrm{B}$. Marconetto, M.P. Babot y N. Oliszewski, pp. 95-125. Museo de Antropología, Facultad de Filosofia y Humanidades, Universidad Nacional de Córdoba, Córdoba.

BONOMO, M; G. POLITIS y J.C. CASTRO 2010. Primeros resultados de las investigaciones arqueológicas en el Delta Superior del Paraná y su contribución al atlas arqueológico de la provincia de Entre Ríos. Folia Histórica del Nordeste, 18: 33-58.

BURKART, R.; N.O. BÁRBARO, R.O. SÁNCHEZ y D.A. GÓMEZ. 1999. Ecoregiones de la Argentina. Administración de
Parques Nacionales (A.P.N.) y Programa Desarrollo Institucional Ambiental (PRODIA), Buenos Aires.

CABRERA, A.L. y A. WILLINK. 1980. Biogeografía de América Latina. Secretaría General de la Organización de los Estados Americanos, Programa Regional de Desarrollo Científico y Tecnológico, Washington D.C.

CAMPOS-TRUJILLO, A.; A AGUIRREPRIETO; G. MUÑOZ-ROMERO; M.A. RODRIGUEZ-VILLA y G. QUINTANAMARTINEZ. 2015. Estudio Palinológico de la flora urbana de la ciudad de Chihuahua, México. Acta Botánica Mexico 113: 111-134.

COLOBIG, M.M.; J.O. SÁNCHEZ y A.F. ZUCOL. 2015 Análisis de macrorrestos vegetales en el sitio arqueológico Los Tres Cerros 1 (Isla las Moras, Victoria, Entre Ríos). Revista del Museo de Antropologia 8: 115-124.

CORTELLA, A. y M. POCHETINO. 1994. Starch grain analysis as a microscopic diagnostic feature in the identification of plant material. Economic Botany 48: 171-181.

CORTEllA, A. y M. POCHETINO. 1995. Comparative morphology of starch of three Andean tubers. Starch 47: 45561.

DE OLIVEIRA FREITAS, F. 2002. Uso de grãos de amido na identificação e análise de materiais arqueológicos vegetais. Boletim de Pesquisa e Desenvolvimento 23: 1-21.

DÍAZ DE LA GUARDIA, C.; G. BLANCA; R.M. NIETO. 1988. Aportación al estudio palinológico de la flora ornamental de la ciudad de granada (España). Fragmenta palynologica baetica. Acta botánica malacitana, pp. 354-362.

ESAU, C. 1976. Anatomía vegetal. Ediciones Omega, Barcelona.

FARICELLI, M.E.; T.A. KRAUS y C. A. BIANCO. 2004. Análisis palinológico de las especies melitófilas de la fami- 
lia Fabaceae del centro de la Argentina. Parte I. Revista FAVE Ciencias Agrarias 3: 13-23.

FAYE, P.F.; A.M. PLANCHUELO y M.L. MOLINELLI. 2002. Relevamiento de la flora apícola e identificación de cargas de polen en el sureste de la provincia de Córdoba, Argentina. Agriscientia 19: 9-30.

FERREIRA A.A.; F.A. AMARAL; I.D.G. DUARTE; P.M. OLIVEIRA; R.B. ALVES; D. SILVEIRA; A.O. AZEVEDO; D.S. RASLAN y M.S.A. CASTRO. 2006. Antinociceptive effect from Ipomoea cairica extract. Journal of Ethnopharmacology 105: 148-153.

HIERONYMUS, J. 1882. Plantae Diaphoricae. Florae Argentinae. Boletin. Académico de. Ciencias 4: 199-589.

KORSTANJE, M.A. y M.P. BABOT. 2005. A microfossil characterization from South Andean economic plants. En Places, people and plants: using phytoliths in Archaeology and Palaeoecology, editado por M. Madella, M.K. Jones y D. Zurro, pp. 41-72. Proceedings of the 4th International Meeting on Phytolith Research, Oxbow Books, Cambridge, UK.

LAHITTE, H.B.; J.A. HURRELL y M.J. BELGRANO. 1998. Plantas medicinales rioplatenses. Edición Lola, Buenos Aires.

LOY, T. 1994. Methods in the analysis of starch residues on prehistoric stone tools. En Tropical Archaeobotany: applications and new developments, editado por J. Hather, pp. 86-114. Routledge, Londres.

MARKGRAF, V. y M.L. D'ANTONI. 1978. Pollen flora of Argentina: Modern Spore and Pollen Types of Pteridophyta, Gymnospermae and Angiospermae. The University of Arizona Press, Tucson.

MEIRA, M.; E. PEREIRA DA SILVA; J.M. DAVIS y J.P. DAVID. 2012. Review of the genus Ipomoea: traditional uses, chemistry and biological activities. Brazilian Journal of Pharmacognosy 22:
682-713.

MULHOLLAND, S. y G. RAPP. 1992. Phytolith Systematics: An Introduction. En Phytolith Systematics, editado por G. Rapp y S. Muholland, pp. 1-13. Plenum Press, New York.

MUÑOZ, F. 1996. Plantas medicinales y aromáticas: estudio, cultivo y procesado. Editorial Aedos, Barcelona.

MUSAUBACH, M.G. 2013. Estudios Arqueobotánicos en sociedades cazadoras-recolectoras de ambientes semiáridos: Análisis de microrrestos vegetales en contextos arqueológicos de Pampa Occidental (Argentina). Tesis doctoral, Facultad de Filosofia y Letras, Universidad de Buenos Aires.

RADLEY, J.A. 1943. Starch and its derivatives. Chapman y Hall Ltd., Londres.

RONDINA, R.V.D.; A.L. BANDONI y J.D. COUSSIO. 2008. Especies medicinales argentinas con potencial actividad analgésica. Dominguezia 24: 47-69.

SÁNCHES-DZIB, Y.A.; S. SOSA-NÁJERA y M.S. LOZANO-GARCÍA. 2009. Morfología Polínica de especies de la selva mediana subperennifolia en la Cuenca del río Candelaria, Campeche. Boletin de la Sociedad Botánica de Mexico 84: 83-104.

VARGAS, A.A.J. 2004. Aportación del análisis de fitolitos, almidones y otros referentes microscópicos al estudio de la prehistoria y arqueológica de las Islas Canarias. Tabona: Revista de prehistoria y de arqueología 12: 69-96.

YANG, X.Y. y L. JIANG. 2010. Starch grain analysis reveals ancient diet at Kuahuqiao site, Zhejiang Province. Chinese Science Bulletin 55: 1150-1156. 